\title{
The Multiple Unit Auction with Variable Supply*
}

\author{
Yvan Lengwiler ${ }^{\dagger}$
}

May 29, 1998

\begin{abstract}
The theory of multiple unit auctions traditionally assumes that the offered quantity is fixed. I argue that this assumption is not appropriate for many applications because the seller may be able and willing to adjust the supply as a function of the bidding. In this paper I address this shortcoming by analyzing a multi-unit auction game between a monopolistic seller who can produce arbitrary quantities at constant unit cost, and oligopolistic bidders. I establish the existence of a subgame-perfect equilibrium for price discriminating and for uniform price auctions. I also show that bidders have an incentive to misreport their true demand in both auction formats, but they do that in different ways and for different reasons. Furthermore, both auction formats are inefficient, but there is no unambiguous ordering among them. Finally, the more competitive the bidders are, the more likely the seller is to prefer uniform pricing over price discrimination, yet increased competition among bidders may or may not enhance efficiency.
\end{abstract}

JEL classification code. D44.

Keywords. multiple unit auction, elastic supply, decreasing valuation, uniform price, price discrimination.

${ }^{*}$ I thank Ian Gale, Daniel Heller, Roger Lagunoff, Marcos Lisboa, Serge Moresi, Athanasios Orphanides, and Kendrew Witt for helpful comments, and Preston McAfee for suggesting a simple example, as well as the participants of the third conference of the Society for the Advancement of Economic Theory 1997 and of seminars at Georgetown and at Stanford University. The views expressed are not neccessarily those of the Board of Governors of the Federal Reserve System or of the Swiss National Bank.

$\dagger$ Board of Governors of the Federal Reserve System and Swiss National Bank.

Please address correspondence to Yvan Lengwiler, Board of Governors of the Federal Reserve System, Division of Monetary Affairs, Mail Stop 71, Washington, DC, 20551, USA, phone (202) 452 2607, email yle@altavista.net 


\section{Introduction}

Auctions are a common form of organizing trade. They are used to allocate precious paintings, oil drilling rights, as well as financial securities. Auction theory studies the properties of equilibria that emerge from different auction rules. The following three questions are the main focus of the theory:

(i) Does the auction game posess an equilibrium?

(ii) How do different auction rules compare in terms of allocative efficiency? and

(iii) What kind of auction would the seller choose (assuming that the seller designs the rules of the auction)?

Traditionally the literature on multiple unit auctions has made two strong assumptions, namely that demand is flat and that supply is fixed. I will argue that both assumptions are not appropriate for many applications. Furthermore, a substantial part of the literature focusses on comparing the uniform price auction with the price discriminating auction, due to the controversy about how the Treasury should auction off its government debt (for instance, Chari and Weber, 1992, and Back and Zender, 1993). In this paper I also restrict attention to these two important formats.

The first assumption of a flat demand function goes back to Vickrey (1962). It states that each bidder demands some fixed quantity $\bar{q}$ up to a price $\bar{p}$, and demands nothing if the price is higher. This "unit-demand assumption" simplifies the analysis considerably because it fixes the quantity an agent demands. The problem is reduced to determining the price he will bid. For many applications, though, the unit-demand assumption is not appropriate. For instance, the most important bidders participating in Treasury bond auctions are banks or brokers. They bid for themselves, as well as on behalf of their customers who have given them buy orders. Even if each individual had a unit-demand function, aggregating the demand functions of all agents represented by a bank or broker will result in a more general, decreasing demand function. Not before very recently some researchers have started to analyze models of uniform price and price discriminating auctions that feature bidders with decreasing demand functions (Noussair, 1995, Engelbrecht-Wiggans and Kahn, 1995a,b, Ausubel and Cramton, 1996, section 5, Tenorio 1997a,b, Draaisma and Noussair, 1997). The general conclusion is that results from models with unit-demand functions do not easily carry over to situations with more general demand functions.

All of these models (except for Hansen, 1988) share the second assumption mentioned above, namely that the supplied quantity is fixed. With this assumption, the seller's decision problem is trivial: he simply picks the highest price that is consistent with selling the given amount. This assumption of a vertical supply schedule seems appropriate for some applications, such as the sale of airport takeoff and landing slots (Gale, 1994), or the auction of the right to supply electricity (von der Fehr and Harbord, 1993). For other applications, the fixed supply assumption is not evident. For instance, a firm that issues new securities to finance its operations has an incentive to adapt the total volume of the issue depending on the demand for the new securities. In fact, one can view the firm's problem as that of a monopolist who operates a constant returns to scale technology when "producing" new 
securities. The cost of issuing an additional unit equals the present value of the stream of payments the security generates. This stream, as well as the firm's discount factor, may be private information of the firm, so that the marginal cost of an additional security may also be private information. Maximizing profit in these circumstances clearly does not imply a fixed supply. Rather, the firm will issue a large amount if bidding is strong (because then finance is cheap), and it will issue only a small amount (or cancel the issue altogether) if bidding is weak. ${ }^{1}$

The introduction of an elastic supply has the potential to alter the answers to the three questions given in the beginning. With regard to question (iii), the seller of a fixed supply simply prefers the auction format that yields the highest average price. If supply is elastic, the average price is not a sufficient criterion anymore, because the sold quantity also affects the seller's profit. With respect to question (ii), if the supply is fixed, efficiency is only a matter of allocating the commodity to those bidders that value it the most. If the supply is variable, however, there is an additional source of inefficiency because the total quantity that is produced and traded may deviate from the social optimum.

This paper addresses both traditional, but restrictive, assumptions of multiple unit auction theory. I characterize the equilibrium of a bidding game between a monopolistic seller (the issuer) with constant but privately observed marginal production costs, and a possibly small set of bidders (the investors), each having a strictly decreasing demand function. First I show that bidders optimally misreport their true valuation in both auction formats [propositions 2 and 5], but they do that in different ways and for different reasons. In the price discriminating auction bidders shade their bids in order to safeguard some of the consumer rent from the apprehension by the seller. In the uniform price auction, bidders shade their bids at high prices, but they overbid at low prices. This fact contrasts the results of models with a fixed supply. In those models, overbidding never occurs in uniform price auctions. Rather, bidders submit less aggressive bids in order to "make room for weaker bidders," so as to reduce the expected price and thereby to increase their consumer rent. This auction format thus promotes an implicit collusion among the bidders. If the supply is elastic, there is no scope for such collusion, because bidding less aggressively only affects the supplied quantity, but does not reduce the expected cut-off price.

Second, both auction formats are inefficient, but the ordering in terms of efficiency loss is ambiguous. The price discriminating auction is inefficient because the bidders misreport their true valuation, and are therefore allocated an inefficient quantity. The uniform price auction is inefficient because, besides the misrepresentation by the bidders, the seller also sets a strictly positive markup, thus driving the totally traded quantity below the efficient amount. In the price discriminating auction, the equilibrium allocation, and thus the amount of inefficiency, is unaffected by the oligopolistic structure of the bidders. In contrast, in the uniform price auction, the misrepresentation vanishes and the markup increases as more bidders join the contest [proposition 3]. Consequently, the more competitive the structure of the bidders is, the more likely the seller is to prefer the uniform price auction over the price discriminating auction. Yet, the social welfare implications of more competition among bidders are ambiguous, because one source of inefficiency (misrepresentation) is substituted

\footnotetext{
${ }^{1}$ Even if the total quantity is announced beforehand, the firm can in principle arbitrarily adjust the effectively issued quantity by allocating an appropriate amount of the securities to itself.
} 
by another one (markup).

Third, I prove that both auction formats considered do have an equilibrium [theorems 1 and 2] in pure strategies. For the price discriminating auction with fixed supply, Menezes and Monteiro (1995) have developed a set of rather restrictive conditions to be imposed on the demand functions that are sufficient and necessary for an equilibrium to exist. Thus, there is no hope for a general existence result for this type of auction. ${ }^{2}$ My existence result therefore provides a resolution of this non-existence problem, and makes the assumption of elastic supply appear even more attractive.

\section{The Model}

Consider a multi-unit auction with one seller and a finite number $n$ of bidders. I will use a price grid as in Nautz (1995). To keep the model tractable I will reduce this grid to the minimum, and assume that there are only two possible prices, $p_{\ell}$ and $p_{h}$, with the convention that $p_{h}>p_{\ell}>0$. The auction is a two stage game, with the bidders submitting their bids in the first stage of the game, and the seller picking a price in the second stage. I look for (pure strategy) subgame-perfect equilibria. To that avail I will solve the model backwards, computing first the seller's best reply, and then solving the bidders' problem.

Assumption 1. Each bidder has some income, $I_{i}$, and a quasi-linear utility function, $u_{i}(q, z):=v_{i}(q)+z$, where $q$ denotes $i$ 's consumption of the commodity we are concerned with, and $z$ is a composite commodity consisting of all the other goods that are of interest to $i$, and whose price we normalize to unity. $v_{i}$, which we call $i$ 's valuation function, is continuously differentiable, monotonically increasing, and strictly concave. Moreover, $v^{\prime}(0)>p_{\ell}$.

Consider the decision problem of a competitive consumer with this utility function, facing a price $p$ for the commodity in question. Let $q_{i}$ denote the number of units he buys, thus the remaining income is $I_{i}-p q_{i}$, and his utility is $v_{i}\left(q_{i}\right)+I_{i}-p q_{i}$. The consumer chooses $q_{i}$ such as to maximize this expression, which gives rise to a demand function $d_{i}$, mapping $p$ to the utility maximizing quantity $q_{i}$. If there is an interior solution, the first-oder condition must be satisfied, i.e. $v_{i}^{\prime}\left(q_{i}\right)-p=0$. Otherwise, there is a corner at $q_{i}=0$. Thus, the demand function is $d_{i}(p):=\max \left\{v_{i}^{\prime-1}(p), 0\right\}$. The assumption that $v_{i}$ is strictly concave implies that $d_{i}$ is strictly decreasing at prices where demand is positive. ${ }^{3}$ Because $v_{i}$ is continuously differentiable, $d_{i}$ is continuous (but not necessarily differentiable). This rules out preferences of the type that yield unit-demand functions. The assumption that $v_{i}^{\prime}(0)>p_{\ell}$ implies that $d_{i}\left(p_{\ell}\right)>0$, so every bidder has some positive demand at the low price. This assumption is not restrictive because if a potential bidder has such a low valuation for the commodity that $d_{i}\left(p_{\ell}\right)=0$ we might just as well drop this bidder from the analysis: He will not participate in the auction anyway.

Note that since bidders are not in a competitive situation, it makes little sense to

\footnotetext{
${ }^{2}$ This is true if we confine ourselves to pure strategy equilibria. Haller and Lengwiler (1998) show, among other things, that an equilibrium in mixed strategies always exists.

${ }^{3}$ More precisely, for all $p$ such that $d_{i}(p)>0$ we have $d_{i}\left(p^{\prime}\right)<d_{i}(p)$ for all $p^{\prime}>p$.
} 
talk about demand functions in this model. ${ }^{4}$ I nevertheless use this term. The demand functions, $d_{i}$, are merely an alternative way of describing the bidders' preferences, $u_{i}$ or $v_{i}$. All the analysis that follows is carried out using the $d_{i}$-functions only. Because preferences are quasi-linear there are no income effects, and maximizing utility $u_{i}$ is equivalent to maximizing the net consumer surplus $\int_{0}^{q} d_{i}^{-1}(\tilde{q}) d \tilde{q}-p q$. As in Menezes and Monteiro (1995), the demand functions are common knowledge among the bidders.

In the first stage of the game, each bidder picks two (non-negative) numbers, $x_{i}$ and $y_{i}$, $i \in\{1, \ldots, n\}$, such that $x_{i} \geqslant y_{i}$, representing the quantity bidder $i$ will buy at the low and at the high price, respectively. For the aggregate bid I write $\Sigma x$ and $\Sigma y$ as a shortcut for $\sum_{i=1}^{n} x_{i}$ and $\sum_{i=1}^{n} y_{i}$, respectively. There is one seller who can produce arbitrary quantities of the commodity at unit $\operatorname{cost} \beta$. $\beta$ is a random variable drawn from a distribution $G$.

Assumption 2. $G(0)=0 . G(\beta)>0$ for all $\beta>0 . G\left(p_{\ell}\right)<G\left(p_{h}\right) . G$ is continuously differentiable and strictly increasing at all $\beta \in\left(0, p_{\ell}\right]$.

This assumption says that density is well defined, strictly positive, and continuous between 0 and $p_{\ell}$, and $p_{\ell}$ and $p_{h}$ are such that there is some mass between them. Note that the differentiability assumption rules out complete information. Complete information requires $G$ to be a step function, jumping from zero to one at the true, commonly known $\beta$.

$G$ is common knowledge, but the realization of $\beta$ is private information to the seller. In the second stage of the game, the seller picks either the low or the high price, or cancels the auction, $\pi \in\left\{p_{\ell}, p_{h}\right.$, cancel $\}$. If the seller picks $p_{h}$, then bidder $i$ receives $y_{i}$; if the seller picks $p_{\ell}$, bidder $i$ receives $x_{i}$ units; if the seller cancels the auction, no bidder receives anything. How much each bidder has to pay depends on the format of the auction (price discrimination or uniform price) and is described later. $\pi$ depends on the information of the seller, which contains his "type" $\beta$ as well as the aggregate bid vector $(\Sigma x, \Sigma y)$, so $\pi$ is in fact a function (or a correspondence if there are multiple best replies), mapping the triple $(\beta, \Sigma x, \Sigma y)$ to either $p_{\ell}$ or $p_{h}$, or to "cancel," depending on which results in the highest profit.

\section{Uniform Price Auction}

In a uniform price auction, every bidder pays the same price for each unit he receives. For instance, if the seller chooses the low price, then bidder $i$ pays $p_{\ell} x_{i}$. The seller's return is $p_{\ell}$ times the amount sold, and the profit is his markup, $p_{\ell}-\beta$, times the amount sold.

The seller chooses either $p_{\ell}$ or $p_{h}$, depending on which price results in higher profits. This will of course depend on this unit $\operatorname{cost} \beta$. It may be that his cost are so high that either price results in a loss. In this case, the seller is free to cancel the auction.

\footnotetext{
${ }^{4}$ I thank Marcos Lisboa for pointing this out to me.
} 
Proposition 1 (equilibrium price). In a subgame-perfect equilibrium of the uniform price auction, the seller's equilibrium strategy is

$$
\pi(\beta, \Sigma x, \Sigma y):= \begin{cases}\text { cancel } & p_{h} \leqslant \beta \\ p_{h} & \beta^{*} \leqslant \beta \leqslant p_{h} \\ p_{\ell} & \beta \leqslant \beta^{*}\end{cases}
$$

with

$$
\beta^{*}:= \begin{cases}\left(p_{\ell} \Sigma x-p_{h} \Sigma y\right) /(\Sigma x-\Sigma y) & \text { if } \Sigma x-\Sigma y>0 \\ 0 & \text { otherwise }\end{cases}
$$

Note that $\beta^{*}$ is in fact a function, mapping $(\Sigma x, \Sigma y)$ to some number. To ease notation I simply write $\beta^{*}$ instead of the more complete $\beta^{*}(\Sigma x, \Sigma y)$.

Proof. First note that the seller can guarantee non-negative profits if and only if $\beta \leqslant p_{h}$. This proves the first row of the formula. Suppose $\Sigma x-\Sigma y>0$. The definition of profit maximization and a few transformations then yield the result for the other two cases,

$$
\begin{aligned}
\pi(\beta, \Sigma x, \Sigma y)=p_{\ell} & \Longleftrightarrow\left(p_{h}-\beta\right) \cdot \Sigma y \leqslant\left(p_{\ell}-\beta\right) \cdot \Sigma x \\
& \Longleftrightarrow p_{h} \Sigma y-p_{\ell} \Sigma x \leqslant \beta \cdot \underbrace{(\Sigma y-\Sigma x)}_{<0} \\
& \Longleftrightarrow \frac{p_{h} \Sigma y-p_{\ell} \Sigma x}{\Sigma y-\Sigma x} \geqslant \beta .
\end{aligned}
$$

$\Sigma x<\Sigma y$ is not possible because by assumption $x_{i} \geqslant y_{i}$ for all $i$. The only remaining case is $\Sigma x=\Sigma y$. Then the seller is always better off choosing the high price (or to cancel the auction) than to choose the low price, because the sold quantity is the same, but the markup is greater with the high price: Setting $\beta^{*}=0$ in that case makes sure that the seller never chooses the low price by assumption 2 .

QED

Proposition 1 characterizes the seller's behavior. He will cancel the auction if and only if $\beta$ (his unit cost) is bigger than the highest possible price. Otherwise, the seller chooses the low price if and only if his unit cost are small enough. What "small enough" means depends on the aggregate bid and is defined by equation (1). Note also that according to this equation this "threshold- $\beta$ " depends on bidder $i$ 's (as well as any other bidders') behavior. This captures the fact that an individual bidder is not a price taker in this model: By changing his bid he can influence the seller's choice of price.

I now turn to the bidders' problem. Bidder $i$ chooses $\left(x_{i}, y_{i}\right)$ so as to maximize the expected consumer rent, taking as given the behavior of his fellow bidders $\left(\Sigma_{j \neq i} x_{j}, \Sigma_{j \neq i} y_{j}\right)$ and nature's randomness $G$. The other $n-1$ bidders behave analogously. Bidder $i$ 's problem is thus formally given by

$$
\begin{aligned}
& \max _{x_{i}, y_{i}} F_{\ell}(\Sigma x, \Sigma y) \cdot L_{i}\left(x_{i}\right)+F_{h}(\Sigma x, \Sigma y) \cdot H_{i}\left(y_{i}\right), \quad \text { with } \\
& L_{i}\left(x_{i}\right):=\int_{0}^{x_{i}} d_{i}^{-1}(q) d q-p_{\ell} x_{i} \quad \text { and } \quad H_{i}\left(y_{i}\right):=\int_{0}^{y_{i}} d_{i}^{-1}(q) d q-p_{h} y_{i},
\end{aligned}
$$

subject to $y_{i} \leqslant x_{i}$. 
$L_{i}\left(x_{i}\right)$ is the consumer rent if the low price is chosen and the bidder is allocated $x_{i}$ units. $H_{i}\left(y_{i}\right)$ is defined analogously. $F_{\ell}(\Sigma x, \Sigma y)$ is the probability that bidder $i$ attaches to the event of the seller's choosing the low price $p_{\ell}$, and similarly for $F_{h}$. Bidder $i$ knows $G$ and, in equilibrium, anticipates $\left(\Sigma_{j \neq i} x_{j}, \Sigma_{j \neq i} y_{j}\right)$, so he can compute $\beta^{*}$ (from proposition 1$)$. The probability that the seller chooses the low price is $G\left(\beta^{*}\right)$, and the probability that he cancels the auction is $1-G\left(p_{h}\right)$. Thus,

$$
F_{\ell}(\Sigma x, \Sigma y)=G\left(\beta^{*}\right) \text { and } \quad F_{h}(\Sigma x, \Sigma y)=G\left(p_{h}\right)-G\left(\beta^{*}\right) .
$$

The bidder's problem is therefore rewritten as

$$
\max _{x_{i}, y_{i}} G\left(\beta^{*}\right) \cdot L_{i}\left(x_{i}\right)+\left(G\left(p_{h}\right)-G\left(\beta^{*}\right)\right) \cdot H_{i}\left(y_{i}\right),
$$

subject to $y_{i} \leqslant x_{i}$.

The following result is helpful in order to characterize the optimal bidding strategy.

\section{Lemma.}

(i) $\partial_{x_{i}} \beta^{*} \geqslant 0$ and $\partial_{y_{i}} \beta^{*}<0 .^{5}$ The first inequality is strict if and only if $\Sigma y>0$.

(ii) $L_{i}\left(x_{i}\right)>H_{i}\left(y_{i}\right)$.

(iii) $0<\beta^{*} \leqslant p_{\ell}$. The second inequality is strict if and only if $\Sigma y>0$.

(iv) $x_{i}>y_{i} \geqslant 0$.

( $i$ ) describes the influence an individual bidder has on the seller's choice. By increasing the bid at the low price, bidder $i$ can increase the probability of the low price's being chosen by the seller. By increasing his bid at the high price, this probability is decreased. $(i v)$ says that each bidder bids strictly more at the low price than at the high price. As a corollary, everyone participates in the auction. Finally, $\beta^{*} \leqslant 0$ means that $\beta$ exceeds $\beta^{*}$ with probability one, so that the seller will never choose the low price. The first inequality in (iii) rules that out in equilibrium. The second inequality in (iii) says that the threshold- $\beta$ is always below $p_{\ell}$. Intuitively this is immediate: If $\beta$ turns out to be weakly greater than $p_{\ell}$, then it cannot possibly be optimal for the seller to choose the low price, because this will cause a loss that can be avoided by choosing the high price or by cancelling the auction. The difference between $p_{\ell}$ and $\beta^{*}$ measures the seller's markup, because if $\beta^{*}<\beta<p_{\ell}$, then the seller could choose the low price without making a loss, but he chooses the more profitable high price. Because demand is strictly decreasing, this reduces the traded quantity below the social optimum, thereby introducing an inefficiency.

Proof. Step 1, "There exists some $i$ with $x_{i}>0$." Because $x_{i} \geqslant y_{i}$ by assumption, $x_{i}=0$ implies $y_{i}=0$, so I need only to establish that $((0,0), \ldots,(0,0))$ is not an equilibrium bidding profile. If $\Sigma y=0$, then any $x_{i}>0$ makes the probability that the seller chooses $\pi=p_{\ell}$ strictly positive. Moreover, by assumption $1, d_{i}\left(p_{\ell}\right)>0$, hence the strategy $\left(d_{i}\left(p_{\ell}\right), 0\right)$ dominates $(0,0)$ for bidder $i$.

\footnotetext{
${ }^{5} \partial_{x_{i}} \beta^{*}$ is the derivative of $\beta^{*}$ with respect to $x_{i}$, and analogously for $\partial_{y_{i}} \beta^{*}$.
} 
Step 2, "If $\Sigma x-\Sigma y>0$, then, $\forall i, \partial_{x_{i}} \beta^{*} \geqslant 0$ and $\partial_{y_{i}} \beta^{*}<0$. The first inequality is strict if and only if $\Sigma y>0$." This is easily computed using (1),

$$
\begin{aligned}
& \partial_{x_{i}} \beta^{*}=\frac{\left(p_{h}-p_{\ell}\right) \Sigma y}{(\Sigma x-\Sigma y)^{2}} \geqslant 0, \\
& \partial_{y_{i}} \beta^{*}=-\frac{\left(p_{h}-p_{\ell}\right) \Sigma x}{(\Sigma x-\Sigma y)^{2}}<0 .
\end{aligned}
$$

The first inequality is strict if and only if $\Sigma y>0$. The second inequality is strict by step 1 .

Step 3, "If $x_{i}>y_{i}$, then $L_{i}\left(x_{i}\right)>H_{i}\left(y_{i}\right)$." Let $\left(x_{i}, y_{i}\right)$ be a strategy such that $L_{i}\left(x_{i}\right) \leqslant$ $H_{i}\left(y_{i}\right)$. Notice that $L_{i}(0)=H_{i}(0)=0$, and $L_{i}(z)>H_{i}(z)$ for all $z>0$ (because $p_{\ell} z<p_{h} z$ ). Note also that $L_{i}(z)$ first increases, reaches its maximum at $z=d_{i}\left(p_{\ell}\right)$, and then decreases. Similarly, if $d_{i}\left(p_{h}\right)>0$, then $H_{i}(z)$ first increases, reaches its maximum at $z=d_{i}\left(p_{h}\right)$, and then decreases again. By assumption, $x_{i} \geqslant y_{i}$, thus $L_{i}\left(x_{i}\right) \leqslant H_{i}\left(y_{i}\right)$ requires either $\left(x_{i}, y_{i}\right)=(0,0)$, or otherwise $x_{i}$ must be greater than $y_{i}$ (because $L_{i}(z)>H_{i}(z)$ for all $z>0$ ) and $x_{i}$ must be such that $L_{i}$ is decreasing at $x_{i}$, that is to say, $L_{i}^{\prime}\left(x_{i}\right)<0$, which requires $x_{i}>d_{i}\left(p_{\ell}\right)$. We will see that this implies a violation of the first-order condition.

Because $x_{i}>y_{i}$ by assumption, the $x_{i}$-component of the solution to the maximization problem is interior, thus the first-order condition must hold. The first-order condition with respect to $x_{i}$ is

$$
\begin{aligned}
& \frac{d}{d x_{i}}\left(G\left(\beta^{*}\right) \cdot L_{i}\left(x_{i}\right)+\left(G\left(p_{h}\right)-G\left(\beta^{*}\right)\right) \cdot H_{i}\left(y_{i}\right)\right)= \\
& \underbrace{G^{\prime}\left(\beta^{*}\right)}_{>0} \cdot \underbrace{\partial_{x_{i}} \beta^{*}}_{>0} \cdot \underbrace{\left(L_{i}\left(x_{i}\right)-H_{i}\left(y_{i}\right)\right)}_{\leqslant 0}+\underbrace{G\left(\beta^{*}\right)}_{>0} \cdot \underbrace{L_{i}^{\prime}\left(x_{i}\right)}_{<0}<0,
\end{aligned}
$$

so the first-order condition is violated. As a consequence, $L_{i}\left(x_{i}\right) \leqslant H_{i}\left(y_{i}\right)$ is not possible in equilibrium.

Step 4, "0 $<\beta^{*}$." By step 1 there exists a bidder $i$ that bids some $x_{i}>0$ in equilibrium. By step $3, L_{i}\left(x_{i}\right)>H_{i}\left(y_{i}\right)$ because $x_{i}>0$. Now suppose $\beta^{*} \leqslant 0$. By (1) this implies that $p_{h} \Sigma y \geqslant p_{\ell} \Sigma x[*]$. By proposition $1, \beta^{*} \leqslant 0$ means that the seller either chooses the high price or cancels the auction, but he will never choose the low price. Equation (3) thus simplifies to

$$
\max _{x_{i}, y_{i}} G\left(p_{h}\right) \cdot H_{i}\left(y_{i}\right) .
$$

Note that $x_{i}$ has no effect on this maximum, so the bidder can in principle arbitrarily increase $x_{i}$, conditional upon not violating [*], without affecting his expected payoff. [*] holds as long as $x_{i} \leqslant \tilde{x}:=\Sigma y \cdot\left(p_{h} / p_{\ell}\right)-\Sigma_{j \neq i} x_{j}$. I will show that it is never optimal for the bidder to set $x \leqslant \tilde{x}$. By increasing $x_{i}$ sufficiently (making it greater than $\tilde{x}$ ), the bidder can violate $[*]$ and thus make $\beta^{*}$ positive again. In fact, because $\beta^{*}$ is a continuous function of $x_{i}$, for every small enough but positive $g$ there exists some $x_{i}>\tilde{x}$ such that $\beta^{*}=g$. In other words, the bidder can choose any arbitrarily small but strictly positive $\beta^{*}$. Then his maximization problem is again given by equation (3) (slightly arranged),

$$
\max _{x_{i}, y_{i}} G\left(\beta^{*}\right) \cdot\left(L_{i}\left(x_{i}\right)-H_{i}\left(y_{i}\right)\right)+G\left(p_{h}\right) \cdot H_{i}\left(y_{i}\right) .
$$


Because $L_{i}\left(x_{i}\right)>H_{i}\left(y_{i}\right)$ by step 3 , and because $G$ is a continuous function by assumption 2 , there exists some small but positive $\beta^{*}$ that also makes $G\left(\beta^{*}\right)$ small but positive, so that the solution to $(3)$ is bigger than the solution to $\left(3^{*}\right)$. That means that it is never optimal for bidder $i$ to let himself be confronted with $\beta^{*} \leqslant 0$. Therefore, he will always set $x_{i}>\tilde{x}$. Consequently, $\beta^{*}$ must be strictly positive in equilibrium.

Step 5, " $\Sigma x-\Sigma y>0$." By proposition 1, $\beta^{*}=0$ if $\Sigma x=\Sigma y$, but this is impossible by step 4 , hence $\Sigma x>\Sigma y$.

I now move on to prove the four items of the lemma. Let me start with item $(i v)$, $x_{i}>y_{i} \geqslant 0$. The second inequality follows from the definition of the strategy space (bidders are not allowed to bid negative quantities). Consider the first inequality then, and consider $\left(x_{i}, y_{i}\right)=(0,0)$ first. Since $\beta^{*}>0$ by step 4 there is some strictly positive probability that the seller will choose the low price by assumption 2 and by proposition 1 . Hence, for all $i$, $\left(d_{i}\left(p_{\ell}\right), 0\right)$ dominates $(0,0)$, so this cannot be a best reply. It remains to be shown that $x_{i}=y_{i}=: z>0$ is not possible. Consider the strategy $(z, z)$ with $z>0$ for some bidder $i$. First of all, this implies $z \geqslant d_{i}\left(p_{h}\right)$, for otherwise $\left(z, d_{i}\left(p_{h}\right)\right)$ dominates $(z, z)$. Secondly, this also implies $z \leqslant d_{i}\left(p_{\ell}\right)$, for otherwise $\left(d_{i}\left(p_{\ell}\right), z\right)$ dominates $(z, z)$. Hence $d_{i}\left(p_{h}\right) \leqslant z \leqslant d_{i}\left(p_{\ell}\right)$. This implies $L_{i}^{\prime}(z) \geqslant 0$ and $H_{i}^{\prime}(z) \leqslant 0$ by assumption 1 . Furthermore, $L_{i}(z)>H_{i}(z)$ because $z>0$. I will show that this implies a violation of the first-order condition.

$\left(x_{i}, y_{i}\right)=(z, z)$, with $z>0$, requires that the derivative of $i$ 's objective function with respect to $y_{i}$ be weakly positive because the constraint $x_{i} \geqslant y_{i}$ binds $y_{i}$ from above. This derivative is

$$
\begin{aligned}
& \frac{d}{d y_{i}}\left(G\left(\beta^{*}\right) \cdot L_{i}\left(x_{i}\right)+\left(G\left(p_{h}\right)-G\left(\beta^{*}\right)\right) \cdot H_{i}\left(y_{i}\right)\right)= \\
& \underbrace{G^{\prime}\left(\beta^{*}\right)}_{>0} \cdot \underbrace{\partial_{y_{i}} \beta^{*}}_{<0} \cdot \underbrace{\left(L_{i}\left(x_{i}\right)-H_{i}\left(y_{i}\right)\right)}_{>0}+\underbrace{\left(G\left(p_{h}\right)-G\left(\beta^{*}\right)\right)}_{>0} \cdot \underbrace{H_{i}^{\prime}\left(y_{i}\right)}_{\leqslant 0},
\end{aligned}
$$

which is negative. Consequently, $(z, z)$ cannot be a best reply.

The remaing items are immediate. Steps 2 and 5 imply $(i)$. (iv) and step 3 imply $(i i)$. Step 4 is the first half of $(i i i)$. The second half is to show that $\beta^{*} \leqslant p_{\ell}$. Rearrange (1) as follows,

$$
\beta^{*}=p_{\ell}-\overbrace{\left(p_{h}-p_{\ell}\right)}^{>0} \frac{\overbrace{\sum y}^{\geqslant 0}}{\underbrace{\sum x-\Sigma y}_{>0 \text { by step } 5}} \leqslant p_{\ell} .
$$

This inequality is strict if and only if $\Sigma y>0$.

QED

We are now in a position to characterize equilibrium bids. The next result says that the bidders present a bid function that is flatter than their true demand function. This makes sense intuitively: This is a game between a monopolist and a bunch of oligopolists. Now suppose - just for the sake of intuition - that there was a continuum of possible prices. Then, the seller, as a monopolist, would choose a markup that maximizes profit. This markup is the larger the less price elastic the demand schedule is. By flattening the bid function compared to the true demand function, the bidders confront the seller with a more elastic schedule, thus decreasing his markup, and increasing their own consumer rent. 
Proposition 2 (equilibrium bids). In a subgame-perfect equilibrium of the uniform price auction, all bidders overstate their true demand at the low price, and understate their true demand at the high price, $x_{i} \geqslant d_{i}\left(p_{\ell}\right)$ and $y_{i} \leqslant d_{i}\left(p_{h}\right)$. In fact, optimal bidding is given by

$$
\begin{aligned}
& x_{i}=d_{i}\left(p_{\ell}-\left(p_{h}-p_{\ell}\right) \frac{\Sigma y}{(\Sigma x-\Sigma y)^{2}}\left(L_{i}\left(x_{i}\right)-H_{i}\left(y_{i}\right)\right) \frac{G^{\prime}\left(\beta^{*}\right)}{G\left(\beta^{*}\right)}\right) \geqslant d_{i}\left(p_{\ell}\right), \\
& y_{i}=d_{i}\left(p_{h}+\left(p_{h}-p_{\ell}\right) \frac{\Sigma x}{(\Sigma x-\Sigma y)^{2}}\left(L_{i}\left(x_{i}\right)-H_{i}\left(y_{i}\right)\right) \frac{G^{\prime}\left(\beta^{*}\right)}{G\left(p_{h}\right)-G\left(\beta^{*}\right)}\right) \leqslant d_{i}\left(p_{h}\right) .
\end{aligned}
$$

The inequality in (6) is strict if and only if $\Sigma y>0$. The inequality in (7) is strict if and only if $d_{i}\left(p_{h}\right)>0$.

Remark: Note that $x_{i}$ and $y_{i}$ appear on both sides of these equations, therefore it is not guaranteed that an equilibrium strategy (and hence an equilibrium) exists. All that this proposition says is that, provided an equilibrium exists, then it satisfies the equations of the proposition. The question of existence will be addressed later in theorem 1 .

Proof. By item $(i v)$ of the lemma the constraint $y_{i} \leqslant x_{i}$ never binds, and the first-order condition is necessary for the $x_{i}$-component.

The first-order condition for (3) with respect to $x_{i}$ (slightly rearranged) is

$$
G\left(\beta^{*}\right) \cdot\left(d_{i}^{-1}\left(x_{i}\right)-p_{\ell}\right)=G^{\prime}\left(\beta^{*}\right) \cdot \partial_{x_{i}} \beta^{*} \cdot\left(H_{i}\left(y_{i}\right)-L_{i}\left(x_{i}\right)\right)
$$

Note that by the item $(i)$ of the lemma and by assumption $2, G^{\prime}\left(\beta^{*}\right)$ is well defined and strictly positive. Then, rewrite (8) as follows,

$$
d_{i}^{-1}\left(x_{i}\right)=p_{\ell}-\overbrace{\underbrace{\prime}\left(\beta^{*}\right)}^{\underbrace{G\left(\beta^{*}\right)}_{>0}} \cdot \underbrace{\partial_{x_{i}} \beta^{*}}_{\geqslant 0} \cdot \underbrace{\left(L_{i}\left(x_{i}\right)-H_{i}\left(y_{i}\right)\right)}_{>0} .
$$

Evaluating $\partial_{x_{i}} \beta^{*}$ as in (4) and applying $d_{i}$ on both sides yields (6). By the lemma, $(i)$ and (ii), the right-hand side of (9) is weakly smaller than $p_{\ell}$. Accordingly, $d_{i}^{-1}\left(x_{i}\right) \leqslant p_{\ell}$, or equivalently (because $d_{i}^{-1}$ is a decreasing function), $x_{i} \geqslant d_{i}\left(p_{\ell}\right)$. If $\Sigma y>0$, then $\partial_{x_{i}} \beta^{*}>0$, and thus $d_{i}^{-1}\left(x_{i}\right)<p_{\ell}$, or $x_{i}>d_{i}\left(p_{\ell}\right)$.

The proof of the other inequality is analogous. I give it here for completeness. If there is an interior solution, then the first-order condition with respect to $y_{i}$ (again slightly rearranged),

$$
d_{i}^{-1}\left(y_{i}\right)=p_{h}+\frac{G^{\prime}\left(\beta^{*}\right)}{G\left(p_{h}\right)-G\left(\beta^{*}\right)} \cdot \partial_{y_{i}} \beta^{*} \cdot\left(H_{i}\left(y_{i}\right)-L_{i}\left(x_{i}\right)\right),
$$

must be satisfied. Evaluating $\partial_{y_{i}} \beta^{*}$ as in (5) and applying $d_{i}$ on both sides yields (7). Given the lemma, $(i)$ and $(i i)$, the right-hand side of (10) is larger than $p_{h}$, thus $d_{i}^{-1}\left(y_{i}\right)>p_{h}$, or equivalently, $y_{i}<d_{i}\left(p_{h}\right)$.

If there is no interior solution there is a corner at $y_{i}=0$, but this requires no special attention here because (7) automatically delivers $y_{i}=d_{i}\left(p_{h}+\right.$ something $)=0$ in this case.

If $d_{i}\left(p_{h}\right)=0$, then there must be a corner, $y_{i}=0$, and the second inequality in (7) is only weak.

QED 
Theorem 1 (existence). The uniform price auction has a subgame-perfect equilibrium.

Proof. I look for subgame-perfect equilibria and therefore solve the game backwards. The solution to the second stage (the seller's optimal behavior) is described in proposition 1, so I will deal in the following with the collapsed game, where the second stage is replaced by the given behavior of the seller (which is known to be optimal). Hence, the seller's strategy $\pi$ will not show up in this proof.

Let $z_{i}$ denote the pair $\left(x_{i}, y_{i}\right)$, and let $z$ be the vector $\left(z_{1}, \ldots, z_{n}\right)$. According to proposition 2 the best reply correspondence $z^{*}$ is defined by a system of $2 n$ equations defining $2 n$ variables as follows,

$$
\begin{aligned}
x_{i}^{*}(z) & :=d_{i}\left(a_{i}(z)\right) \quad \text { and } \quad y_{i}^{*}(z):=d_{i}\left(b_{i}(z)\right) \text { with } \\
a_{i}(z) & :=p_{\ell}-\left(p_{h}-p_{\ell}\right) \frac{\Sigma y}{(\Sigma x-\Sigma y)^{2}}\left(L_{i}\left(x_{i}\right)-H_{i}\left(y_{i}\right)\right) \frac{G^{\prime}\left(\beta^{*}\right)}{G\left(\beta^{*}\right)} \\
b_{i}(z) & :=p_{h}+\left(p_{h}-p_{\ell}\right) \frac{\Sigma x}{(\Sigma x-\Sigma y)^{2}}\left(L_{i}\left(x_{i}\right)-H_{i}\left(y_{i}\right)\right) \frac{G^{\prime}\left(\beta^{*}\right)}{G\left(p_{h}\right)-G\left(\beta^{*}\right)} .
\end{aligned}
$$

Let $z_{i}^{*}(z):=\left(x_{i}^{*}(z), y_{i}^{*}(z)\right)$ and $z^{*}(z):=\left(z_{1}^{*}(z), \ldots, z_{n}^{*}(z)\right)$. An equilibrium is a fixed point of $z^{*} . d_{i}$ is continuous by assumption $1 . a_{i}$ and $b_{i}$ are continuous because $G$ is continuously differentiable by assumption 2. Hence, $z_{i}^{*}$ (and therefore $z^{*}$ ) are continuous functions.

Now, pick some arbitrary number $M$ such that $\max \left\{d_{i}\left(p_{\ell}\right): i=1, \ldots, n\right\}<M<\infty$, and define $\bar{x}_{i}^{*}(z):=\min \left\{x_{i}^{*}(z), M\right\}, \bar{z}^{*}(z):=\left(\bar{x}^{*}(z), y^{*}(z)\right)$, and $S:=\chi_{i=1}^{n}\left[d_{i}\left(p_{\ell}\right), M\right] \times$ $\left[0, d_{i}\left(p_{h}\right)\right]$. By proposition $2, x_{i}^{*}(z) \geqslant d_{i}\left(p_{\ell}\right)$ and $y_{i}^{*}(z) \leqslant d_{i}\left(p_{h}\right)$, hence $\bar{z}^{*}$ is a continuous function whose range is contained in $S$. By restricting the domain of $\bar{z}^{*}$ to $S$ we therefore get a continuous function from the compact and convex set $S$ into itself. Brouwer's Theorem thus applies and guarantees the existence of a fixed point, $\tilde{z}=\bar{z}^{*}(\tilde{z})$. This quasi-equilibrium $\tilde{z}$ is an equilibrium if $\forall i \bar{x}_{i}^{*}(\tilde{z})=x_{i}^{*}(\tilde{z}) \leqslant M$, but one can always find a large enough, but finite, $M$ such that this is the case, so an equilibrium exists.

QED

Note that the equilibrium is always inefficient, for two reasons. First, the seller uses a strictly positive markup. Hence, unlike in a competitive market, the price is greater than the marginal cost of production. Second, the bidders misreport their true valuation, and are therefore allocated inefficient quantities.

Example: unit-demand. It is rather difficult to actually compute equilibria using even rather simple demand and distribution functions (such as linear demand and uniform distribution). With a unit-demand function, however, computation is simple. This example also demonstrates that having a strictly decreasing demand function is necessary for the result that bidders misreport their demand.

Consider the case of a bidder whose demand is a step function with a single step,

$$
d(p):= \begin{cases}q & \text { if } p \leqslant \bar{p} \\ 0 & \text { otherwise. }\end{cases}
$$

With such a demand function this auction is similar to a single-unit auction, because a bidder either buys $q$ or nothing. Suppose also that $p_{\ell}<\bar{p}<p_{h}$. Accordingly, $d\left(p_{\ell}\right)=q$ and 
$d\left(p_{h}\right)=0$. Since $x$ and $y$ will always be in the range of $d$ by proposition 2 , and the range in this simple case consists just of two points, 0 and $q$, and $x$ cannot exceed $y$, there are three possible equilibrium bids to consider: $(x, y) \in\{(0,0),(q, 0),(q, q)\}$.

If $(x, y)=(0,0)$, then $(L, H)=(0,0)$. As a consequence, $y=d\left(p_{h}\right)=0$, which is okay, but $x=d\left(p_{\ell}\right)=q$, contradicting the equilibrium property of $(0,0)$.

If $(x, y)=(q, q)$, then $(L, H)=\left(\left(\bar{p}-p_{\ell}\right) q,-\left(p_{h}-\bar{p}\right) q\right)$, and $L-H=\left(p_{h}-p_{\ell}\right) q>0$ (in accordance with the lemma). As a consequence, $x=d\left(p_{\ell}-\right.$ something $)=q$, which is okay, but $y=d\left(p_{h}+\right.$ something $)=0$, contradicting the equilibrium property of $(q, q)$.

Finally, consider $(x, y)=(q, 0)$. In this case, $(L, H)=\left(\left(\bar{p}-p_{\ell}\right) q, 0\right)$, and $x=d\left(p_{\ell}-\right.$ something $)=q$, and $y=d\left(p_{h}\right)=0$, both of which are compatible with the equilibrium property of $(q, 0)$. Hence, an equilibrium exists and bidders will bid according to their true valuation. The bids are not distorted only because the demand function is flat. With a downward sloping demand, bidders would misreport their demand.

The next result characterizes how the equilibrium changes as the competition among bidders increases, meaning individual bidders become more and more negligible.

Proposition 3 (limit as individual bidders become negligible). Consider an $r$-fold replica of the set of bidders, i.e. there are $r$ copies of each bidder, so that in sum there are rn bidders. As $r$ increases, the bids of the individual bidders converge to their true demand. Formally, $\lim _{r \rightarrow \infty} x_{i}=d_{i}\left(p_{\ell}\right)$ and $\lim _{r \rightarrow \infty} y_{i}=d_{i}\left(p_{h}\right)$.

Proof. Let $\left(x_{i j}, y_{i j}\right)$ denote the strategy of the $j$ th copy of bidder $i . \Sigma x$ and $\Sigma y$ now denote the average aggregate bids per set of bidders, $\Sigma x:=\left(\Sigma_{j} \Sigma_{i} x_{i j}\right) / r$, and similarly for $\Sigma y$. In the replica game, the derivative of $\beta^{*}$ with respect to $x_{i j}$, given in (4), becomes

$$
\partial_{x_{i j}} \beta^{*}=\left(p_{h}-p_{\ell}\right) \cdot \frac{r \Sigma y}{r^{2}(\Sigma x-\Sigma y)^{2}} .
$$

This derivative vanishes as $r$ grows indefinitely. Hence, the right-hand side of (9) converges to $p_{\ell}$, and in the limit, $d_{i j}{ }^{-1}\left(x_{i j}\right)=p_{\ell}$, or equivalently, $x_{i j}=d_{i j}\left(p_{\ell}\right)$. An analogous argument applies to $y_{i j}$.

QED

¿From Nautz (1995) it is known that price taking bidders report their true demand in a uniform price auction. Proposition 3 establishes that this is also where the behavior of increasingly negligible bidders converges to: "almost negligible" bidders report their demand "almost truthfully."

Note that, from a welfare theoretic point of view, the increased competition among bidders has an ambiguous effect: As competition increases, misrepresentation of demand vanishes, which is good because it reduces the misallocation, but the seller's markup increases, driving the produced quantity farther away from the social optimum. Thus, perfect competition among the bidders is not necessarily better than an oligopolistic structure of bidders. 


\section{Price Discriminating Auction}

The strategy sets of the players of a price discriminating auction game are the same as in a uniform price auction, but payoffs are different. If the seller picks the high price, then the bidder receives $y_{i}$ units and pays $p_{h}$ for each unit. If the seller chooses the low price, then the bidder receives $x_{i}$ units, but he pays $p_{h}$ for the first $y_{i}$ units, and he pays the lower price

$p_{\ell}$ only for the additional $x_{i}-y_{i}$ units. His total bill will therefore be $p_{h} y_{i}+p_{\ell}\left(x_{i}-y_{i}\right)$. If the auction is cancelled, then no units are sold and the bidders pay nothing.

Proposition 4 (equilibrium price). In a price discriminating auction, the seller's best reply is given by

$$
\pi(\beta):= \begin{cases}\text { cancel } & p_{h} \leqslant \beta \\ p_{h} & p_{\ell} \leqslant \beta \leqslant p_{h} \\ p_{\ell} & \beta \leqslant p_{\ell}\end{cases}
$$

Proof. Define $A:=\left(p_{h}-\beta\right) \cdot \Sigma y$ and $B:=\left(p_{\ell}-\beta\right) \cdot(\Sigma x-\Sigma y)$. In a price discriminating auction, profit equals $A$ if the seller picks the high price, and it equals $A+B$ if the seller picks the low price. If $\beta$ is greater than $p_{h}$, then profit is negative, no matter what price the seller chooses ( $A$ and $B$ are negative), so in this situation it is best for him to cancel the auction. If $\beta$ is between $p_{h}$ and $p_{\ell}$, then $A$ is positive, but $B$ is negative, hence it is optimal to choose the high price. Finally, if $\beta$ is smaller than $p_{\ell}$, then both, $A$ and $B$, are positive, and thus it is best to choose the low price.

QED

This proposition says that the seller's behavior depends only on his unit cost. It is independent of the bids. The intuition for this is immediate: The marginal revenue of selling more equals $\pi$, and marginal cost is $\beta$. Hence, profit maximization requires $\pi$ to equal $\beta$. The formula in the proposition is the analogue of this intuition for a finite price grid. This also implies that the seller's behavior gives no rise to inefficiencies: He always sets the cut-off price equal to the marginal cost (or as closely as the price grid allows that).

Proposition 5 (equilibrium bids). In a subgame-perfect equilibrium of the price discriminating auction, bidders report their true demand at the low price, but understate their true demand at the high price. This distortion is independent of the number of bidders. Formally,

$$
x_{i}=d_{i}\left(p_{\ell}\right), \quad \text { but } \quad y_{i}=d_{i}\left(p_{h}+\left(p_{h}-p_{\ell}\right) \frac{G\left(p_{\ell}\right)}{G\left(p_{h}\right)-G\left(p_{\ell}\right)}\right) \leqslant d_{i}\left(p_{h}\right) .
$$

The inequality is strict if and only if $d_{i}\left(p_{h}\right)>0$.

Proof. As before, let $F_{\ell}$ denote the probability of the low price being chosen by the seller, and similarly for $F_{h}$. From proposition $4 \mathrm{I}$ have

$$
F_{\ell}=G\left(p_{\ell}\right) \quad \text { and } \quad F_{h}=G\left(p_{h}\right)-G\left(p_{\ell}\right) .
$$


Note that these probabilities are independent of the bidders' behavior, which makes this problem simpler than the uniform price auction. The expected consumer rent is given by

$$
F_{h} \cdot\left(\int_{0}^{y_{i}} d_{i}^{-1}(q) d q-p_{h} y_{i}\right)+F_{\ell} \cdot\left(\int_{0}^{x_{i}} d_{i}^{-1}(q) d q-p_{h} y_{i}-p_{\ell}\left(x_{i}-y_{i}\right)\right) .
$$

Bidder $i$ chooses $\left(x_{i}, y_{i}\right)$ such as to maximize (12). The first-order condition with respect to $x_{i}$ is

$$
F_{\ell} \cdot\left(d_{i}^{-1}\left(x_{i}\right)-p_{\ell}\right)=0
$$

which implies

$$
x_{i}=d_{i}\left(p_{\ell}\right) .
$$

Thus, the bidder reports his true demand at the low price.

The first-order condition with respect to $y_{i}$ is slightly more complicated,

$$
F_{h} \cdot\left(d_{i}^{-1}\left(y_{i}\right)-p_{h}\right)-F_{\ell} \cdot\left(p_{h}-p_{\ell}\right)=0 .
$$

By assumption 2, both, $F_{\ell}$ and $F_{h}$, are strictly positive. As a consequence,

$$
y_{i}=d_{i}\left(p_{h}+\left(p_{h}-p_{\ell}\right) \frac{F_{\ell}}{F_{h}}\right) \leqslant d_{i}\left(p_{h}\right) .
$$

Thus, the bidder understates his true demand at the high price. By assumption 1, the second inequality is strict if $d_{i}\left(p_{h}\right)>0$.

QED

The result reported in proposition 5 is not new. It was basically found by Nautz (1995), based on Smith's (1966) model. They assume that the price is an exogenous random variable. As a consequence, no bidder can affect the distribution of the price and they behave as pricedistribution takers. Here, the price is endogenous, because it is the seller who decides upon it. Yet, as shown in proposition 4 , the seller has a dominant strategy in the price-discriminating auction: His choice of price is independent of the bidding. Therefore, the price distribution is still exogenous to the bidders in the sense that even a large bidder (or even all bidders together, were they to collude) cannot affect it. For this reason, large bidders behave just like small bidders do and rationally take the price-distribution as given. As a corollary, Nautz' result applies also to the case of few, non-negligible bidders, which is just what proposition 5 says. In fact, even Nautz' proof goes through basically unaltered.

Suppose - just for the sake of generating intuition for this result - that there was no price grid and any positive number could be a price. Truth-telling then means that a bidder submits his true demand function. He will be allocated the quantity that corresponds to his demand at the cut-off price chosen by the seller, and he will pay the area under his demand function up to this quantity. As a result, price discrimination is perfect and the seller extracts the whole consumer surplus from the bidder. The bidder will be just indifferent between this outcome and not participating at all. Hence, this strategy cannot be optimal. By reducing his bid somewhat the bidder runs the risk of being allocated a smaller quantity than what he truly wants, but this strategy reduces his cost and saves at least some of the consumer rent. 
Having said that, we conclude that the price discriminating auction is also inefficient. If the seller's marginal cost are such that he chooses the high price in equilibrium, than bidders will be allocated smaller quantities than what is socially optimal in the sense that their marginal valuation exceeds the price paid, thus leaving room for some unexploited Pareto improvement.

I now move on to the question of existence of an equilibrium.

Theorem 2 (existence and uniqueness). The price discriminating auction has a unique subgame-perfect equilibrium.

Proof. The seller has a strictly dominant strategy that does not depend on the bids. Because the $d_{i}$-functions are strictly decreasing, (11) defines a unique best reply for the bidders as well. Each bidder's best reply is a dominant strategy because it only depends on exogenous factors, such as $d_{i}$ or $G$, but not on the behavior of other bidders. Combining all these best replies to a strategy profile is therefore trivially a fixed point of the best reply map. Hence, a combination of equilibrium strategies, $\pi(\cdot)$ and $\left(\left(x_{1}, y_{1}\right), \ldots,\left(x_{n}, y_{n}\right)\right)$, and therefore an equilibrium, exists and is unique.

QED

This result sharply contrasts with Menezes and Monteiro (1995). They find that a price discriminating auction typically has no equilibrium, unless restrictive assumptions are imposed on the demand functions. Two differences between their model and mine should be kept in mind. First, they work with a simultaneous move game and look for Nash equilibria. I analyze an extensive form game in which the bidders move first, and look for subgameperfect equilibria. Second, they assume that the quantity that is to be sold is fixed. As a result, the bids associated with the lowest accepted price have to be rationed (if their sum exceeds the remaining quantity). This rationing introduces a discontinuity that jeopardizes existence. In contrast, I assume a horizontal supply schedule, and therefore no need for rationing. As a consequence, my model does not exhibit this discontinuity, and existence is guaranteed.

Note that assumptions 1 and 2 can be weakened considerably without jeopardizing the validity of propositions 4 and 5 and of theorem 2 . All we need is that $d_{i}$ be continuous and strictly decreasing for all $i$ and $G\left(p_{\ell}\right)<G\left(p_{h}\right)$. G need not be continuous. In particular, complete information does not need to be ruled out, that is to say, $G(\beta)=0$ for $\beta<\bar{\beta}$, $G(\beta)=1$ for $\beta \geqslant \bar{\beta}$, and $p_{\ell}<\bar{\beta} \leqslant p_{h}$ is compatible with these results.

Note also an important difference between the equations that characterize an equilibrium under both auction formats. Proposition 3 establishes that, in the uniform price auction, the more competitive the bidders are, the less distorted the bids will be. In the limit, as they approach price taking, bidders report their true demand. Nothing comparable is at work in the price discriminating auction: bidders shade their bids not to influence the behavior of the seller (which, in fact, depends only on $\beta$ only and is independent of the bids), but to save some of the consumer rent, which would be zero (with a continuum of possible prices) if they reported their true demand. We are thus led to the following statement.

Observation. In the price discriminating auction, the equilibrium strategies are independent of the oligopolistic structure of the bidders. 
So the behavior of the bidders is unaffected by their oligopolistic structure. In other words, "few large bidders" (or even the coalition of all bidders, were they to cooperate) behave the same way as "many small bidders" would. Therefore, the equilibrium allocation of the price discriminating auction, and thus its inefficiency, is unaffected by the intensity of competition among bidders.

Remember that in the uniform price auction the misrepresentation vanishes as more bidders join the contest (proposition 3). Hence, the more competitive the bidders are, the less price elastic is the aggregate bid function the seller faces, and the greater are his profit maximizing markup and his profits. Consequently, the more competitive the structure of the bidders is, the more likely the seller is to prefer the uniform price auction over the price discriminating auction.

\section{Conclusion}

Despite the widespread use of auctions, the theory of multiple unit auctions is still far from complete. Two rather simple formats for multiple unit auctions that are widely used on financial markets are the uniform price auction and the price discriminating auction. This paper focuses on these two formats.

Typically it is assumed that there is some fixed amount of the commodity that is to be auctionned off. I argue that this assumption is not always appropriate, especially for applications to the auctions of newly issued securities, because the effectively issued quantity can in principle be adjusted by the issuer on the spot. Assuming a perfectly elastic supply instead alters the conventional results of the literature dramatically.

First, with an elastic supply, both types of auctions do have an equilibrium in pure strategies, as opposed to the price discriminating auction with fixed supply, which does not in general have a pure strategy equilibrium (Menezes and Monteiro, 1995). Second, in contrast to the literature assuming price-taking behavior (Smith, 1966), in this model bidders misreport their true valuation not only in the price discriminating, but also in the uniform price auction. Furthermore, the computation of equilibrium strategies is much easier for price discriminating auctions than for uniform price auctions, thus reversing the "strategic simplicity argument" that has been made in favor of uniform pricing (Nautz, 1995, Nautz and Wolfstetter, 1997). Third, for the uniform price auction with fixed supply it was found that bidders try to "make room for weaker bidders" (e.g., Noussair, 1995). Just the opposite is true with elastic supply: there is overbidding in equilibrium at low prices.

\section{References}

Ausubel, L. M. and Cramton, P. C. (1996). "Demand Reduction and Inefficiency in MultiUnit Auctions." University of Maryland, Department of Economics, Working Paper \#96-07.

Back, K. and Zender, J. F. (1993). "Auctions of Divisible Goods: On the Rationale for the Treasury Experiment." The Review of Financial Studies 6(4), 733-764. 
Chari, V. V. and Weber, R. J. (1992). "How the U.S. Treasury Should Auction its Debt." Federal Reserve Bank of Minneapolis Quarterly Review 16(4), 3-12.

Draaisma, T. and Noussair, Ch. (1997). "Optimal Bidding in a Uniform Price Auction with Multi-Unit Demand." Economics Letters 56, 157-162.

Engelbrecht-Wiggans, R. and Kahn, Ch. M. (1995a). "Multi-Unit Auctions with Uniform Prices." Working Paper \#95-0125, University of Illinois at Urbana-Champaign, forthcoming in Economic Theory.

Engelbrecht-Wiggans, R. and Kahn, Ch. M. (1995b). "Multi-Unit Pay-Your-Bid Auctions with Variable Awards." Working Paper \#95-0142, University of Illinois at UrbanaChampaign, forthcoming in Games and Economic Behavior.

von der Fehr, N. H. M., and Harbord, D. (1993). "Spot Market Competition in the UK Electricity Industry." The Economic Journal 103, 531-546.

Gale, I. (1994). "Competition for Scarce Inputs: The Case of Airport Takeoff and Landing Slots." Federal Reserve Bank of Cleveland Economic Review 30(2), 18-25.

Haller, H. and Lengwiler, Y. (1998). "A Discrete Model of Discriminatory Price AuctionsAn Alternative to Menezes-Monteiro." Finance and Economics Discussion Series 199808, Board of Governors of the Federal Reserve System, Washington, DC.

Hansen, R. G. (1988). "Auctions with Endogenous Quantity." Rand Journal of Economics 19(1), 44-58.

Menezes, F. M. and Monteiro, P. K. (1995). "Existence of Equilibrium in a Discriminatory Price Auction." Mathematical Social Sciences 30, 285-292.

Nautz, D. (1995). "Optimal Bidding in Multi-Unit Auctions with Many Bidders." Economics Letters 48, 301-306.

Nautz, D. and Wolfstetter, E. (1997). "Bid Shading and Risk Aversion in Multi-Unit Auctions with Many Bidders." Economics Letters 56(2), 195-200.

Noussair, Ch. (1995). "Equilibria in a Multi-Object Uniform Price Sealed Bid Auction with Multi-Unit Demands." Economic Theory 5(2), 337-351.

Smith, V. L. (1966). "Bidding Theory and the Treasury Bill Auction: Does Price Discrimination Increase Bill Prices?" Review of Economics and Statistics 48, 141-146.

Tenorio, R. (1997a). "On Strategic Quantity Bidding in Multiple Unit Auctions." The Journal of Industrial Economics 45(2), 207-217.

Tenorio, R. (1997b). "Multiple Unit Auctions with Strategic Price-Quantity Decisions." Forthcoming in Economic Theory.

Vickrey, W. (1962). "Auctions and Bidding Games." In Recent Advances in Game Theory, Princeton University Press. Reprinted in Public Economics-Selected Papers by William Vickrey, ed. by R. Arnott et al., chapter 4, Cambridge University Press, 1994. 